\title{
Porous silicon nitride spacers versus PEEK cages for anterior cervical discectomy and fusion: clinical and radiological results of a single-blinded randomized controlled trial
}

\author{
Mark P. Arts ${ }^{1}\left(\mathbb{D} \cdot\right.$ Jasper F. C. Wolfs $^{1} \cdot$ Terry P. Corbin $^{2}$
}

Received: 19 October 2016/Revised: 19 March 2017/Accepted: 27 March 2017 /Published online: 5 April 2017

(C) The Author(s) 2017. This article is an open access publication

\begin{abstract}
Purpose Anterior cervical discectomy with fusion is a common procedure for treating radicular arm pain. Polyetheretherketone (PEEK) plastic is a frequently used material in cages for interbody fusion. Silicon nitride is a new alternative with desirable bone compatibility and imaging characteristics. The aim of the present study is to compare silicon nitride implants with PEEK cages filled with autograft harvested from osteophytes.

Methods The study is a prospective, randomized, blinded study of 100 patients with 2 years follow-up. The primary outcome measure was improvement in the Neck Disability Index. Other outcome measures included SF-36, VAS arm pain, VAS neck pain, assessment of recovery, operative characteristics, complications, fusion and subsidence based on dynamic X-ray and CT scan.

Results There was no significant difference in NDI scores between the groups at 24 months follow-up. At 3 and 12 months the NDI scores were in favor of PEEK although the differences were not clinically relevant. On most follow-up moments there was no difference in VAS neck and VAS arm between both groups, and there was no statistically significant difference in patients' perceived recovery during follow-up. Fusion rate and subsidence were similar for the two study arms and about $90 \%$ of the implants were fused at 24 months.

Conclusions Patients treated with silicon nitride and PEEK reported similar recovery rates during follow-up. There
\end{abstract}

Mark P. Arts

m.arts@haaglandenmc.nl

1 Department of Neurosurgery, Haaglanden Medical Center, PO Box 432, 2501 CK The Hague, The Netherlands

2 Corbin \& Company, Maple Grove, MN, USA was no significant difference in clinical outcome at 24 months. Fusion rates improved over time and are comparable between both groups.

Keywords Anterior cervical discectomy and fusion . Silicon nitride · PEEK · Randomized controlled trial . Herniated disc

\section{Introduction}

Anterior cervical discectomy and fusion (ACDF) is a common treatment for a cervical radicular syndrome producing persistent arm pain with or without neck pain. The choices for anterior cervical fusion are many, and a recent literature review has described the lack of clarity on the best procedure options for ACDF [1]. To maintain or enlarge the disc space height and maintain lordosis a block of autograft is frequently inserted into the disc space [2, 3]. However, graft harvest for cervical interbody fusion can be associated with pain at the iliac crest harvest site [1].

Interbody fusion cages made of metal, plastic and most recently ceramic materials have been shown to have advantages over autograft bone blocks: (1) using a cage provides strength to support a larger axial load than bone; (2) the cage can be filled with locally harvested autograft (from osteophytes) or bone graft substitutes, eliminating the need for harvest from the iliac crest, and finally, (3) using a cage saves time in the procedure, reduces blood loss and reduces post-operative pain compared with obtaining graft from the iliac crest.

Despite a paucity of data supporting choice of cage materials [4], plastic cages made from polyetheretherketone (PEEK) have become a popular method for stabilizing the disc space after ACDF [5-8]. PEEK as a biomaterial 
has some attractive properties and some liabilities [9]. It has a modulus of elasticity similar to bone. PEEK is hydrophobic and develops a mild fibrous tissue reaction around implants. While it is radiolucent and causes no distortion of X-ray, CT or MR images, the lucency makes it difficult to evaluate cage position and fusion. This problem is partially overcome by inserting radiopaque metal markers into PEEK cages.

In order to address the deficiencies of autograft blocks, implant manufacturers have evaluated additional materials including ceramics. Ceramic materials are attractive because they are generally biocompatible and have desirable imaging properties. The toughest and most stable ceramic is silicon nitride $\left(\mathrm{Si}_{3} \mathrm{~N}_{4}\right)$. Silicon nitride cages have been tested extensively in cadavers, demonstrating desirable imaging characteristics [10], and in animals demonstrating biocompatibility and bacteriostatic properties [11, 12]. Amedica Corporation (Salt Lake City, UT, USA) has designed spacers made from an outer shell of dense silicon nitride and a center filled with a microporous form of the same material (Valeo $\mathrm{C}^{\mathrm{CsC}}$ silicon nitride spacers) (Fig. 1). In a sheep model, these spacers demonstrated robust bone ingrowth resulting in fusion [13].

Because PEEK cages have become the gold standard for cervical interbody fusion, the CAncellous Structure

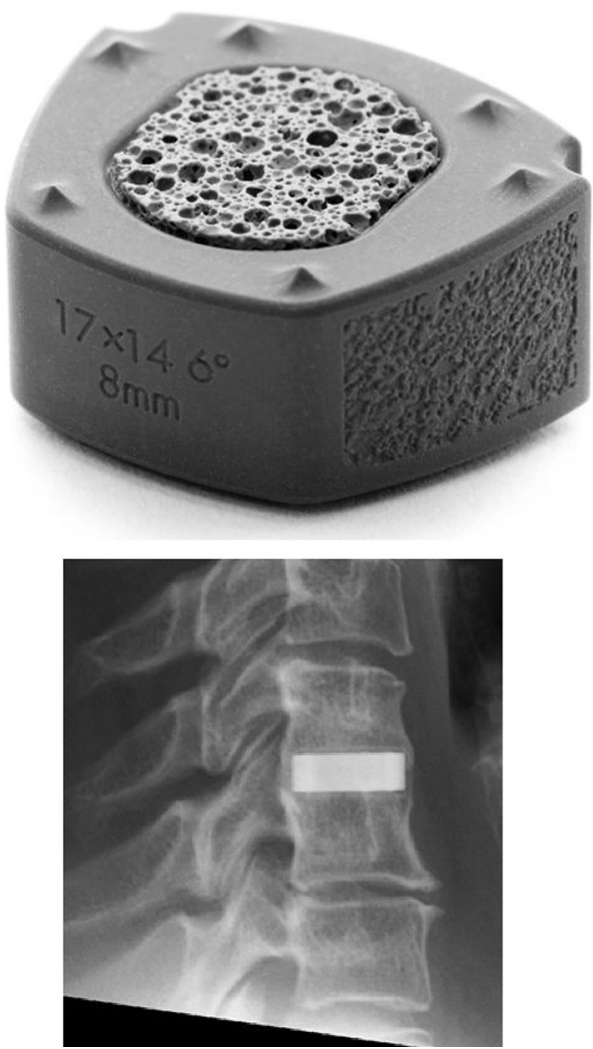

Fig. 1 Valeo $\mathrm{C}^{\mathrm{CsC}}$ silicon nitride cervical interbody fusion device used in the CASCADE trial with radiographic characteristics
Ceramic Arthrodesis DEvice (CASCADE) trial was designed to compare stand-alone silicon nitride spacers filled with microporous silicon nitride to PEEK cages filled with autograft harvested from osteophytes. This article reports the clinical and radiological outcome of patients treated with silicon nitride versus PEEK and is the first paper of human outcomes using silicon nitride cages.

\section{Materials and methods}

The study protocol has been published in detail previously [14] and a summary is available on the clinicaltrials.gov website (ClinicalTrials.gov Identifier: NCT01511445). The trial is also registered in the Netherlands (NL 36013.098.11). Briefly, the study was designed as a prospective, single-blind randomized controlled comparison of single-level ACDF using Valeo $\mathrm{C}^{\mathrm{CsC}}$ silicon nitride spacers versus PEEK cages. Initial analysis was performed when all patients reached 1 year follow-up, and final analysis was conducted when all patients had 2 years follow-up. The study was approved by the Medical Ethics Committee of Southwest Holland. Informed consent was obtained from all individual participants included in the study. The patients were treated by two neurosurgeons of Haaglanden Medical Center, The Hague, Netherlands (MA and JW).

The inclusion and exclusion criteria are similar to other ACDF randomized trials using PEEK cages as control groups [15, 16] (Table 1). Patients were included if they presented with radicular symptoms in one or both arms indicating a single nerve root involvement with or without medullary symptoms and with or without neck pain. The diagnosis of a herniation with or without osteophytes at a single level was confirmed radiographically. Exclusion criteria included previous cervical surgery, severe kyphosis at the involved level, neck pain only without radicular or medullary symptoms, metabolic disease, neoplasms, cervical trauma, spinal anomalies, severe mental or psychiatric disease, and inadequate Dutch language.

Randomization (1:1 ratio of silicon nitride spacers to PEEK cages) was carried out in the operating room after anesthesia was induced by opening sequentially numbered, sealed envelopes that were prepared by the data manager. The randomization order was determined using a random number generator to produce random blocks of four, six and eight numbers. Patients were blinded to the group allocation during the first year of follow-up.

\section{Surgical procedure}

A standard right-side approach was made after confirming the level on fluoroscopy. A standard anterior discectomy 
Table 1 Inclusion and exclusion criteria

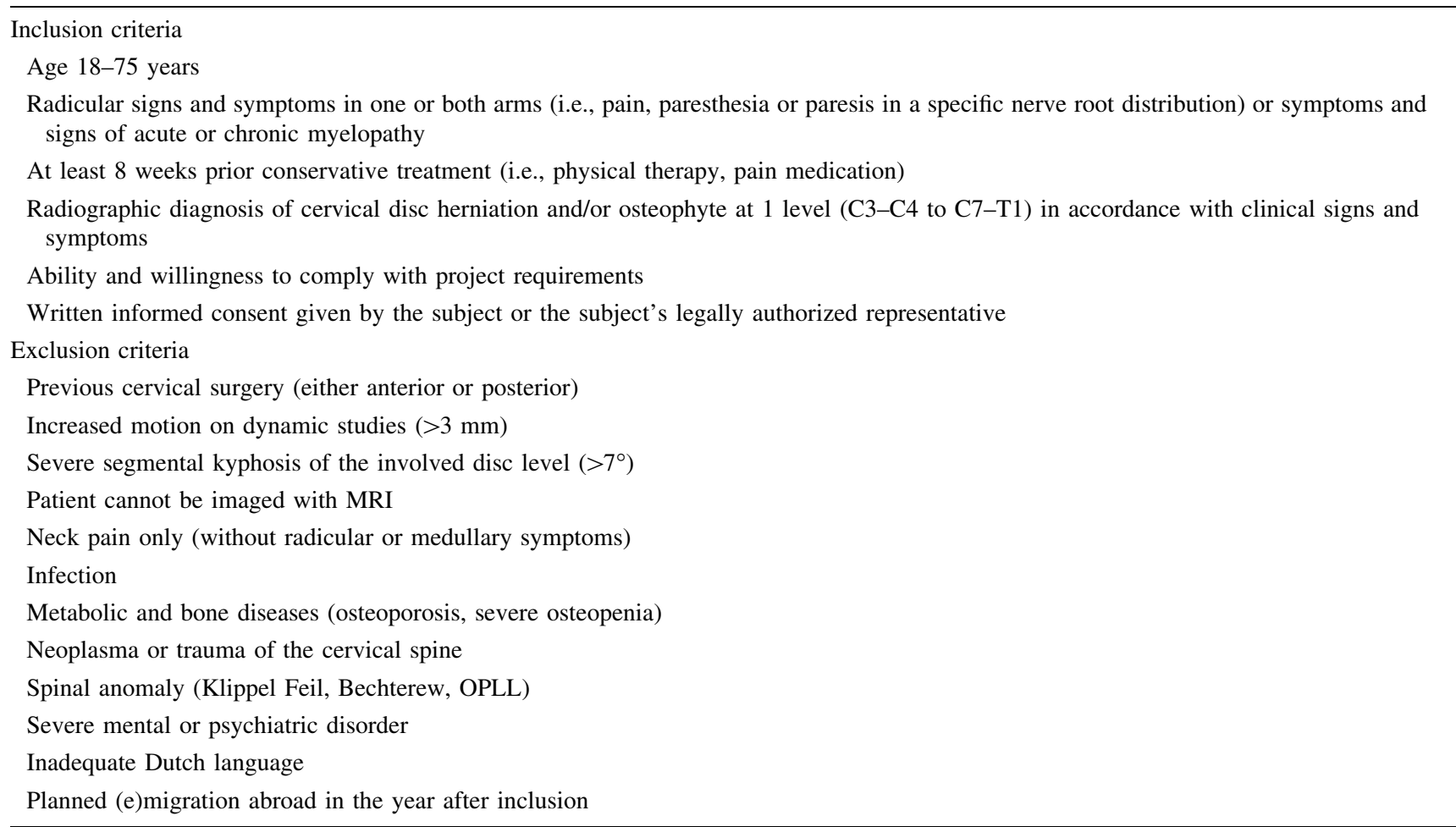

was performed with the aid of loupe magnification or operating microscope, depending on the preference of the surgeon. After complete decompression, the allocated spacer or cage was implanted. Silicon nitride spacers (Amedica Corporation, Salt Lake City, UT, USA) were wetted with patient blood from the operative field but had no other filler. PEEK cages (Medicrea Manta, Lyon, France) were filled with local autograft obtained from osteophytes at the operative level. No patients had autograft or any other substance placed outside the spacers or cages, and no patients had supplemental fixation such as plates and screws. The spacers and cage sizes were chosen to incorporate the largest possible footprint; the typical cage was $17 \mathrm{~mm}$ wide by $14 \mathrm{~mm}$ in depth. The silicon nitride implants had parallel superior and inferior surfaces, while the PEEK cages had $3^{\circ}$ of lordosis. After surgery, all patients were mobilized as soon as possible without a collar.

\section{Primary non-inferiority hypothesis}

The primary effectiveness hypothesis, as stated in the Clinical Trial Protocol, is that there is no difference in Neck Disability Index (NDI) improvement between the two study arms. This hypothesis was formulated for testing clinical non-inferiority based on the Blackwelder approach [17]. In the Blackwelder approach, the null hypothesis is that the investigational device is clinically significantly worse than control by an amount (at least) equal to an a priori selected non-inferiority margin. The minimal clinically important difference (MCID) for the NDI is 7.5 points out of 50 , or $15 \%$ when the scale is standardized to a range from 0 to 100 [18]. Therefore, the primary null hypothesis is that the mean improvement for the investigational device is smaller (i.e., less negative) than the mean improvement for the control device by an amount equal to or exceeding 15 out of the 100-point scale.

\section{Sample size determination}

The sample size was calculated with $90 \%$ power to detect a difference in NDI improvement equal to the MCID of $15 \%$, the non-inferiority margin. The sample size calculation used the improvement amount and standard deviation observed in a previous study of carbon fiber-reinforced PEEK cages [19]. The calculated sample size was 46 patients in each group, and an additional 4 patients were added to each group to allow $8 \%$ for loss to follow-up.

\section{Outcome measures}

The primary outcome measure is improvement in the NDI which has been translated into Dutch and validated for the population of the Netherlands [20, 21]. Secondary outcome measures were the $100-\mathrm{mm}$ visual analog scale (VAS) for arm pain and neck pain [22], the 7-point Likert self-rating 
scale for perceived recovery in which "complete recovery" and "almost complete recovery" are defined as good outcome [23], and the generic health survey on the Short Form-36 (SF-36) [24, 25]. Adverse events, reoperations, and surgical complications were also recorded.

\section{Radiological assessment}

At each follow-up time point, four plane films were collected (standing anterior-posterior, lateral, flexion and extension radiographs). A thin-slice CT scan was also collected at the 6 months follow-up. In addition, quantitative and qualitative motion analysis using Medical Metrics, Inc. (MMI, Houston, TX, USA) software [26] allowed measurement of rotation on flexion-extension films with an accuracy of $\pm 1^{\circ}$. Images for each follow-up time point were provided on compact disks for analysis by MMI. Fusion for this study was defined as rotation $\leq 4^{\circ}$ and $\leq 1.25 \mathrm{~mm}$ translation on flexion-extension films. The dynamic X-ray and CT at 6 months follow-up were also used to measure cage migration and subsidence. Radiologists quantified bone bridging around implants, bone bridging through the PEEK cage and lucencies around both types of implant.

\section{Statistical analysis}

The statistical analysis plan was modified from the published protocol to meet FDA recommendations for a prospective study prior to the completion of data collection. Pre-operative data were analyzed for all randomized patients. The Fisher's exact test (two-sided) was used for categorical variables, while the Wilcoxon rank sum test was used for continuous variables. Missing values in the non-inferiority analysis were conservatively filled using the last value carried forward method.

\section{Results}

Between December 2011 and October 2013, 104 of 390 eligible patients were enrolled in the randomized trial. Four patients were censored because of protocol violations (surgery by non-investigators). All presented data are performed on the intention-to-treat analysis of 100 patients. The complete study patient accountability flowchart is presented in Fig. 2. Fifty-two patients were randomized for silicon nitride and 48 patients for PEEK. Baseline characteristics for both groups were similar (Table 2). The only statistical difference was seen in the level of treatment with significantly more patients in the silicone nitrate group having surgeries in the lower cervical spine $\mathrm{C} 6 \mathrm{C} 7$ and C7T1. Over $40 \%$ of the study subjects were smokers. There
Neck Disability Index (NDI)

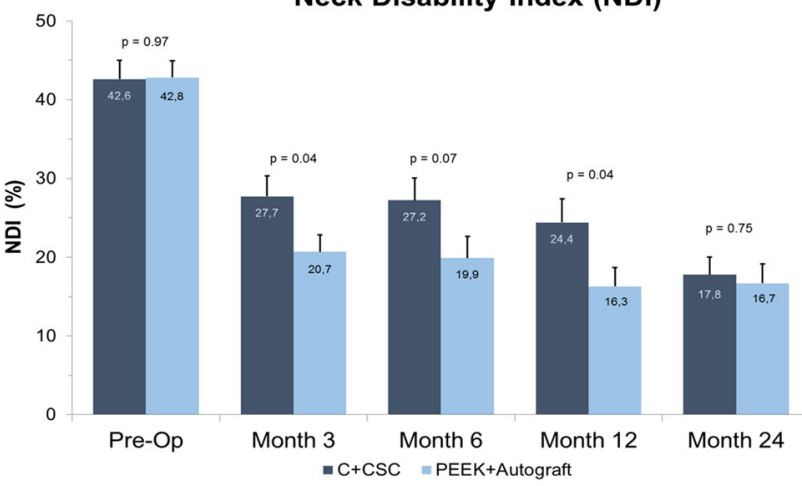

Fig. 2 Neck and Disability Index scores during follow-up of 24 months. At 3 and 12 months there were small significant differences in favor of PEEK, although not clinically relevant

were no crossovers or allocation errors. At 24 months follow-up, data from 96 patients were available.

\section{Clinical outcome}

\section{Primary outcome measure}

Patients in both study arms showed large improvements in NDI scores during the 24-month follow-up. Patients treated with PEEK had significantly better improvement at 3 months and 12 months, although these differences were less than the MCID for the NDI of 7.5 points. There was no significant difference in NDI score at 2 years (Fig. 2).

\section{Other outcome measures}

VAS arm pain and VAS neck pain improved in both groups, although there was a significant difference in arm pain at 12 months and neck pain at 3 months, both favoring PEEK. These differences did not reach the MCID of $20 \mathrm{~mm}$ and were therefore not clinically relevant. Of all SF-36 domains, only Bodily Pain at 3 months follow-up had a statistically significant difference, favoring PEEK patients. The patients' reported perceived recovery improved during the follow-up moments; $78 \%$ of the silicon nitride patients and $88 \%$ of the PEEK patients reported good outcome at 24 months $(p>0.05)$. All secondary outcome measures are illustrated in Table 3.

\section{Complications and re-operations}

The surgical procedures and hospital stay were uneventful for almost all patients (Table 4). There was no difference in operative time, estimated blood loss, or length of stay between the two groups. Two PEEK patients and one silicon nitride patient had incidental durotomies which were repaired intra-operatively and had no permanent sequelae. 
Table 2 Baseline demographics of 100 analyzed patients $(4$ were excluded because of protocol violation)

\begin{tabular}{llll}
\hline Demographics & Silicon nitride $(N=52)$ & PEEK $(N=48)$ & $p$ value \\
\hline Female gender $(\%)$ & $23(44 \%)$ & $23(48 \%)$ & 0.84 \\
Mean age in years (range) & $53.3(34-74)$ & $49.4(28-67)$ & 0.11 \\
Symptoms & & & \\
Radicular pain only & 48 & 39 & \\
Medullary only & 2 & 7 & 0.03 \\
Combined & 3 & 5 & \\
Symptomatic level & & & \\
C3C4 & $1(2 \%)$ & $1(2 \%)$ & \\
C4C5 & $0(0 \%)$ & $6(13 \%)$ & \\
C5C6 & $30(58 \%)$ & $30(63 \%)$ & $10(21 \%)$ \\
C6C7 & $19(37 \%)$ & $1(2 \%)$ & 0.55 \\
C7T1 & $2(4 \%)$ & $19(40 \%)$ & 0.18 \\
Smoking & $24(46 \%)$ & $28.6(20.6-75)$ & 0.96 \\
Mean BMI (range) & $26.7(19.4-39.1)$ & $42.8 \pm 14.9$ & 0.56 \\
NDI ( \pm SD) & $42.6 \pm 17.1$ & $55.3 \pm 24.5$ & 0.55 \\
SF-36 physical function & $59.4 \pm 17.9$ & $34.5 \pm 20.9$ & \\
SF-36 bodily pain & $37.7 \pm 23.5$ & &
\end{tabular}

$S F$ Short Form, $S D$ standard deviation, BMI Body Mass Index
Nine silicon nitride and eight PEEK patients had transient dysphagia, with or without hoarseness. One patient in each group had recurrent symptomatic nerve root compression at the index level because of substantial subsidence. In both cases, the device was replaced by an allograft block and the patient had supplemental fixation with a plate and screws. One silicon nitride patient had a deep wound infection requiring debridement without spacer removal and the patient was treated with antibiotics. During the follow-up period of 2 years, nine patients had revision surgery at the adjacent level whereby disc herniations or osteophytes were removed; six in the silicon nitride group and three patients in the PEEK group. The average time from the index surgery to the secondary adjacent level surgery was 12.6 months for silicon nitride patients and 11.3 months for PEEK cage patients.

\section{Fusion and subsidence}

Based on the MMI analysis of flexion/extension motion $\leq 4^{\circ}$, there was no significant difference in fusion rate between silicon nitride and PEEK. In both groups the fusion rate increased over time and improved from $66 \%$ at 3 months follow-up, to $90 \%$ at 24 months follow-up for both groups (Fig. 3). At 6 months follow-up, 26 patients treated with silicon nitride and 27 patients treated with PEEK showed some degree of subsidence at flexion/extension images and CT. The mean subsidence was $1.48 \mathrm{~mm}$ for silicon nitride and $1.59 \mathrm{~mm}$ for PEEK, and the difference was not statistically significantly different (Table 5).

\section{Discussion}

The CASCADE trial was designed to compare microporous silicon nitride as a spacer material versus the gold standard of PEEK filled with autograft. It was modeled after ongoing trials comparing anterior discectomy with ACDF and decompression plus cervical artificial discs $[15,16]$. In those trials, the ACDF procedure used PEEK cages filled with a beta tricalcium phosphate synthetic graft material. The use of local autograft in the CASCADE trial raises the threshold for non-inferiority because autograft produces have a higher fusion rate [27]. Based on the CASCADE trial, patients treated with silicon nitride and patients treated with PEEK cages reported similar recovery rates and improvement in NDI during follow-up with similar fusion rates. However, some intermediate results were in favor of PEEK, but these differences did not reach the level of the minimal clinically important difference and therefore had no clinical relevance.

The main source of pain relief in the procedure of anterior cervical discectomy is decompression of the nerve root by removing the herniated disc and/or osteophytes. Numerous studies on ACDF have demonstrated satisfactory clinical and radiological results in the majority of patients $[1,8,28-30]$. Even in the older population ACDF surgery will result in a significant gain in health-state utility [31]. Adding an interbody implant to the anterior discectomy procedure is primarily to stabilize the height and angulation of the disc space. Loss of height could lead to recurrent stenosis of the foramen and recurrence of radicular pain. Even empty cages are associated with 
Table 3 Treatment effect of primary and secondary outcome during follow-up period

\begin{tabular}{|c|c|c|c|}
\hline & Silicon nitride & PEEK & $p$ value \\
\hline \multicolumn{4}{|c|}{ Neck Disability Index } \\
\hline 3 months & $27.7 \pm 18.5$ & $20.7 \pm 14.5$ & 0.04 \\
\hline 6 months & $27.2 \pm 19.2$ & $19.9 \pm 17.8$ & 0.07 \\
\hline 12 months & $24.4 \pm 20.6$ & $16.3 \pm 16.4$ & 0.04 \\
\hline 24 months & $17.8 \pm 15.2$ & $16.7 \pm 16.2$ & 0.75 \\
\hline \multicolumn{4}{|l|}{ VAS arm } \\
\hline 3 months & $26.4 \pm 27.7$ & $28.7 \pm 31.7$ & 0.72 \\
\hline 6 months & $29.4 \pm 32.4$ & $21.5 \pm 28.5$ & 0.24 \\
\hline 12 months & $33.0 \pm 31.9$ & $20.5 \pm 26.3$ & 0.04 \\
\hline 24 months & $27.4 \pm 28.4$ & $17.3 \pm 21.7$ & 0.06 \\
\hline \multicolumn{4}{|l|}{ VAS neck } \\
\hline 3 months & $32.2 \pm 27.8$ & $29.5 \pm 26.3$ & 0.65 \\
\hline 6 months & $33.2 \pm 31.0$ & $20.4 \pm 23.2$ & 0.03 \\
\hline 12 months & $26.3 \pm 24.9$ & $22.4 \pm 26.8$ & 0.48 \\
\hline 24 months & $18.6 \pm 23.6$ & $17.9 \pm 21.1$ & 0.88 \\
\hline \multicolumn{4}{|c|}{ SF-36 physical functioning } \\
\hline 3 months & $68.6 \pm 24.3$ & $73.5 \pm 22.4$ & 0.33 \\
\hline 6 months & $73.3 \pm 24.1$ & $74.9 \pm 25.0$ & 0.77 \\
\hline 12 months & $75.7 \pm 23.8$ & $79.3 \pm 21.9$ & 0.45 \\
\hline 24 months & $78.4 \pm 21.7$ & $77.6 \pm 27.5$ & 0.88 \\
\hline \multicolumn{4}{|c|}{ SF-36 bodily pain } \\
\hline 3 months & $54.8 \pm 24.5$ & $66.4 \pm 21.1$ & 0.02 \\
\hline 6 months & $57.2 \pm 25.6$ & $67.0 \pm 25.1$ & 0.07 \\
\hline 12 months & $63.4 \pm 25.4$ & $67.3 \pm 25.8$ & 0.46 \\
\hline 24 months & $73.4 \pm 24.0$ & $71.8 \pm 25.3$ & 0.76 \\
\hline \multicolumn{4}{|c|}{ Surgeons perceived recovery } \\
\hline 3 months & 68.0 & 83.0 & 0.10 \\
\hline 6 months & 67.4 & 77.8 & 0.35 \\
\hline 12 months & 70.2 & 80.4 & 0.38 \\
\hline 24 months & 80.4 & 86.7 & 0.57 \\
\hline \multicolumn{4}{|c|}{ Patient perceived recovery } \\
\hline 3 months & 58.3 & 65.2 & 0.53 \\
\hline 6 months & 57.8 & 70.5 & 0.27 \\
\hline 12 months & 58.7 & 76.1 & 0.12 \\
\hline 24 months & 78.3 & 88.1 & 0.26 \\
\hline
\end{tabular}

improvement in pain and disability and remarkable high fusion rates are documented [29, 32].

A recently published randomized controlled trial on acrylic cages versus PEEK cages documented significant clinical and radiological improvement in favor of acrylic cages [28]. The study suggests more cost effectiveness of acrylic implants compared to PEEK, although it is limited by small patient numbers and short-term follow-up. Therefore, in our opinion, the development of new implant technologies is better understood as long as the ultimate and optimal implant has not been defined yet.
In our trial, patients reported similar recovery rates during follow-up regardless of the type of implant. With composite clinical success defined as at least 15-point improvement of NDI (out of 100), fusion determined on dynamic X-rays, and no revision surgery at the index level, $62 \%$ of silicon nitride and $69 \%$ of PEEK achieved composite clinical success at 2 years $(p=0.64)$. At 2 years follow-up, there was no difference in revision surgery of the index level between silicon nitride and PEEK. However, patient treated with silicon nitride were operated more frequently at the adjacent level $(N=6)$ than patients who had implanted PEEK $(N=3)$. The complaints of these patients at follow-up visits and their perceived recovery scores were most likely influenced by the development of adjacent segment symptoms. In all cases, these patients had evidence of degeneration at the adjacent level, but no related symptoms at the time of the index procedure. Possibly, the initial clinical presentation was already based on symptomatology of both levels which could also explain difference in intermediate outcome measures. In addition, the silicon nitride cages were parallel in design while the PEEK cages had $3^{\circ}$ of lordosis. It has been hypothesized that the parallel cages might present a theoretical disturbance of the sagittal balance which could explain the early presentation of adjacent level disease in the silicon nitride patients.

Microporous materials to fill cages are an attractive concept since no autograft harvest or additional expense for cage filler materials is needed. Trabecular metal made from porous tantalum has received extensive testing, but has not fared well as a stand-alone cage material because it lacks strength (leading to fragmentation) and its high radioopacity and metallic distortion interferes with imaging [33]. Silicon nitride spacers, however, did not suffer from these problems. There were no device integrity issues and imaging was not affected by the silicon nitride product.

Animal studies [11] of silicon nitride implants have shown that there is no foreign body encapsulation as is noted with PEEK cages [9]. This attribute apparently makes the material more bacteriostatic. In our study, one infection of a silicon nitride spacer was successfully debrided without spacer removal, resulting in a successful clinical outcome and fusion. While infections are rare and therefore difficult to study, the bacteriostatic properties of silicon nitride implants may allow patients to recover from inadvertent wound contamination without implant removal. This possibility will only be proven by collection of a large amount of patient data through a registry or meta-analysis.

The design of the CASCADE trial had several limitations. The moderate sample size restricted the amount of subgroup analysis that could be performed. The selection of PEEK cages with autograft harvested from osteophytes as a control group is not the most widely used control in ACDF studies; 
Table 4 Operative characteristics with complications

\begin{tabular}{llll}
\hline & Silicon nitride $(N=52)$ & PEEK $(N=48)$ & $p$ value \\
\hline Operative characteristics & & & \\
Operative time, min (range) & $42.0(25-65)$ & $40.6(30-60)$ & 0.41 \\
Blood loss, ml (range) & $107(10-600)$ & $109(10-800)$ & 0.95 \\
Mean hospital stay, days (range) & $1.1(1-3)$ & $1.0(1-2)$ & 0.17 \\
Complications & 9 & 8 & 0.70 \\
Transient dysphagia & 1 & 1 & 1.00 \\
Cage subsidence with revision surgery & 1 & 0 & 1.00 \\
Infection with cage removal & 6 & 3 & 0.49 \\
Adjacent level surgery & & & \\
\hline
\end{tabular}

There were no statistically significant differences with respect to operation time, blood loss, hospital stay, and complications

Fusion Rate

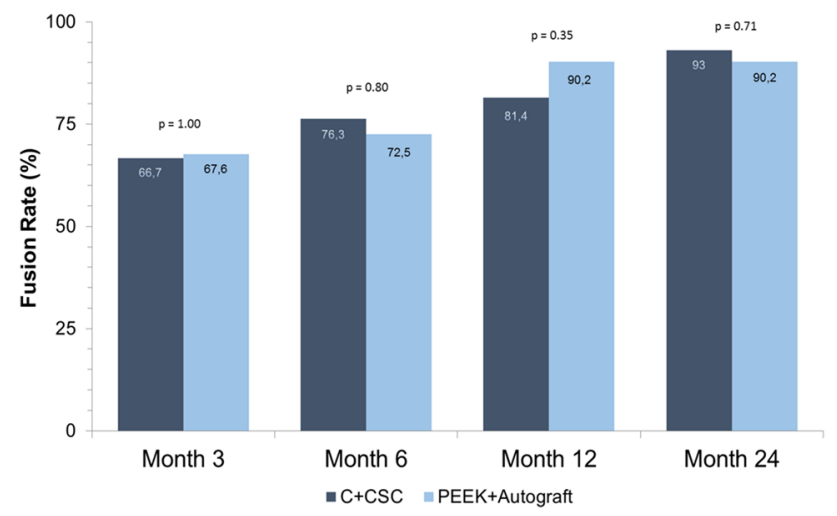

Fig. 3 Fusion rate defined as rotation $\leq 4^{\circ}$ and $\leq 1.25 \mathrm{~mm}$ translation on flexion-extension films on dynamic X-ray. In both groups the fusion rate increased over time and there was no significant difference between both groups

Table 5 Subsidence measured on CT and X-ray at 6 months followup

\begin{tabular}{llll}
\hline $\begin{array}{l}\text { Subsidence } \\
(\mathrm{mm})\end{array}$ & $\begin{array}{l}\text { Silicon nitride } \\
(N=46)\end{array}$ & $\begin{array}{l}\text { PEEK } \\
(N=46)\end{array}$ & $p$ value \\
\hline 0 & 20 & 19 & \\
1 & 3 & 3 & \\
2 & 11 & 8 & \\
3 & 7 & 12 & \\
4 & 4 & 2 & \\
$>4$ & 1 & 2 & 0.33 \\
\hline
\end{tabular}

There was no significant difference in the presence of subsidence between both groups

many US studies incorporate allograft blocks with supplemental fixation as the control group. However, the CASCADE clinical trial represents the worst-case scenario because autograft results in higher fusion rates than allograft. Finally, the study was supported financially by the company Amedica that is the manufacturer of silicon nitride. Every effort was made to eliminate bias in the study design, protocol, and management of the study. Independent Clinical Research Organization (CRO) managed the study together with the principal investigator's institution, the radiographic measurements were also performed by an independent organization employing their radiologists and yet another independent unit was used to perform statistical analysis. With those precautions, the authors have implemented reasonable procedure to minimize bias.

In conclusion, patients with symptomatic herniated disc or spondylosis treated with anterior cervical discectomy and interbody fusion using silicon nitride or PEEK reported similar recovery rates. There was no significant difference in clinical outcome at 24 months, although some intermediate outcome measures were in favor of PEEK but did not reach the level of clinical relevance. Fusion rates of both implants improve over time and no significant difference was seen regarding fusion and subsidence. The primary hypothesis of similarity in NDI improvement between silicon nitride and PEEK has been proven.

Acknowledgements The authors want to thank the sponsor Amedica Corporation for funding the trial. They also want to thank Ditte Varkevisser, Robin van Zijl and Irene van Beelen for their hard work in making this trial possible.

\section{Compliance with ethical standards}

Study funding The study was funded by Amedica Corporation. The study investigators and data manager were compensated for their time by the sponsor.

Conflict of interest The authors MA and JW receive research grants from Zimmer-Biomet, EIT and Intrinsics. MA and JW are consultants for Zimmer-Biomet and Silony and they receive royalties from EIT.

Ethical approval All procedures performed in studies involving human participants were in accordance with the ethical standards of the institutional and/or national research committee and with the 1964 Helsinki declaration and its later amendments or comparable ethical standards. 
Open Access This article is distributed under the terms of the Creative Commons Attribution 4.0 International License (http://crea tivecommons.org/licenses/by/4.0/), which permits unrestricted use, distribution, and reproduction in any medium, provided you give appropriate credit to the original author(s) and the source, provide a link to the Creative Commons license, and indicate if changes were made.

\section{References}

1. Jacobs W, Willems PC, Kruyt M, van Limbeek J, Anderson PG, Pavlov P, Bartels R, Oner C (2011) Systematic review of anterior interbody fusion techniques for single- and double-level cervical degenerative disc disease. Spine (Phila Pa 1976) 36(14):E950-E960

2. Cloward RB (1958) The anterior approach for removal of ruptured cervical disks. J Neurosurg 15(6):602-617

3. Smith GW, Robinson RA (1958) The treatment of certain cervicalspine disorders by anterior removal of the intervertebral disc and interbody fusion. J Bone Joint Surg Am 40-A(3):607-624

4. Kersten RF, van Gaalen SM, de Gast A, Oner FC (2015) Polyetheretherketone (PEEK) cages in cervical applications: a systematic review. Spine J 15(6):1446-1460

5. Celik SE, Kara A, Celik S (2007) A comparison of changes over time in cervical foraminal height after tricortical iliac graft or polyetheretherketone cage placement following anterior discectomy. J Neurosurg Spine 6(1):10-16

6. Hee HT, Kundnani V (2010) Rationale for use of polyetheretherketone polymer interbody cage device in cervical spine surgery. Spine J 10(1):66-69

7. Kulkarni AG, Hee HT, Wong HK (2007) Solis cage (PEEK) for anterior cervical fusion: preliminary radiological results with emphasis on fusion and subsidence. Spine J 7(2):205-209

8. Lied B, Roenning PA, Sundseth J, Helseth E (2010) Anterior cervical discectomy with fusion in patients with cervical disc degeneration: a prospective outcome study of 258 patients (181 fused with autologous bone graft and 77 fused with a PEEK cage). BMC Surg 10:10

9. Toth JM, Wang M, Estes BT, Scifert JL, Seim HB III, Turner AS (2006) Polyetheretherketone as a biomaterial for spinal applications. Biomaterials 27(3):324-334

10. Anderson M, Bernero J, Brodke D (2008) Medical imaging characteristics of silicon nitride ceramic: a new material for spinal arthroplasty implants. Paper presented at the 8th Annual Spine Arthroplasty Society Global Symposium on Motion Preservation Technology, Miami

11. Gorth DJ, Puckett S, Ercan B, Webster TJ, Rahaman M, Bal BS (2012) Decreased bacteria activity on $\mathrm{Si}(3) \mathrm{N}(4)$ surfaces compared with PEEK or titanium. Int J Nanomed 7:4829-4840

12. Webster TJ, Patel AA, Rahaman MN, Sonny BB (2012) Antiinfective and osteointegration properties of silicon nitride, poly(ether ether ketone), and titanium implants. Acta Biomater 8(12):4447-4454

13. Anderson $\mathrm{MC}$, Olsen $\mathrm{R}$ (2010) Bone ingrowth into porous silicon nitride. J Biomed Mater Res A 92(4):1598-1605

14. Arts MP, Wolfs JF, Corbin TP (2013) The CASCADE trial: effectiveness of ceramic versus PEEK cages for anterior cervical discectomy with interbody fusion; protocol of a blinded randomized controlled trial. BMC Musculoskelet Disord 14:244

15. Arts MP, Brand R, van den Akker E, Koes BW, Peul WC, The NEtherlands Cervical Kinematics (NECK) trial (2010) Cost-effectiveness of anterior cervical discectomy with or without interbody fusion and arthroplasty in the treatment of cervical disc herniation; a double-blind randomised multicenter study. BMC Musculoskelet Disord 11:122
16. Bartels RH, Donk R, van der Wilt GJ, Grotenhuis JA, Venderink D (2006) Design of the PROCON trial: a prospective, randomized multi-center study comparing cervical anterior discectomy without fusion, with fusion or with arthroplasty. BMC Musculoskelet Disord 7:85

17. Blackwelder WC (1982) "Proving the null hypothesis" in clinical trials. Control Clin Trials 3(4):345-353

18. Carreon LY, Glassman SD, Campbell MJ, Anderson PA (2010) Neck Disability Index, short form-36 physical component summary, and pain scales for neck and arm pain: the minimum clinically important difference and substantial clinical benefit after cervical spine fusion. Spine J 10(6):469-474

19. Peolsson A (2007) Investigation of clinically important benefit of anterior cervical decompression and fusion. Eur Spine $\mathrm{J}$ 16(4):507-514

20. Vernon H, Mior S (1991) The Neck Disability Index: a study of reliability and validity. J Manipulative Physiol Ther 14(7):409-415

21. Vos CJ, Verhagen AP, Koes BW (2006) Reliability and responsiveness of the Dutch version of the Neck Disability Index in patients with acute neck pain in general practice. Eur Spine J 15(11):1729-1736

22. Collins SL, Moore RA, McQuay HJ (1997) The visual analogue pain intensity scale: what is moderate pain in millimetres? Pain 72(1-2):95-97

23. Bombardier C (2000) Outcome assessments in the evaluation of treatment of spinal disorders: summary and general recommendations. Spine (Phila Pa 1976) 25(24):3100-3103

24. Brazier JE, Harper R, Jones NM, O'Cathain A, Thomas KJ, Usherwood T, Westlake L (1992) Validating the SF-36 health survey questionnaire: new outcome measure for primary care. BMJ 305(6846):160-164

25. Ware JE Jr, Sherbourne CD (1992) The MOS 36-item short-form health survey (SF-36). I. Conceptual framework and item selection. Med Care 30(6):473-483

26. Taylor M, Hipp JA, Gertzbein SD, Gopinath S, Reitman CA (2007) Observer agreement in assessing flexion-extension X-rays of the cervical spine, with and without the use of quantitative measurements of intervertebral motion. Spine J 7(6):654-658

27. Epstein NE (2012) Iliac crest autograft versus alternative constructs for anterior cervical spine surgery: pros, cons, and costs. Surg Neurol Int 3(Suppl 3):S143-S156

28. Farrokhi MR, Nikoo Z, Gholami M, Hosseini K (2017) Comparison between acrylic cage and polyetheretherketone (PEEK) cage in single-level anterior cervical discectomy and fusion: a randomized clinical trial. Clin Spine Surg 30:34-46

29. Shiban E, Gapon K, Wostrack M, Meyer B, Lehmberg J (2016) Clinical and radiological outcome after anterior cervical discectomy and fusion with stand-alone empty polyetheretherketone (PEEK) cages. Acta Neurochir (Wien) 158(2):349-355

30. Chagas H, Domingues F, Aversa A, Vidal Fonseca AL, de Souza JM (2005) Cervical spondylotic myelopathy: 10 years of prospective outcome analysis of anterior decompression and fusion. Surg Neurol 64(Suppl 1):S1-S5

31. Chotai S, Parker SL, Sielatycki JA, Sivaganesan A, Kay HL, Wick JB, McGirt MJ, Devin CJ (2016) Impact of old age on patient-report outcomes and cost utility for anterior cervical discectomy and fusion surgery for degenerative spine disease. Eur Spine J. doi:10.1007/s00586-016-4835-3

32. Pechlivanis I, Thuring T, Brenke C, Seiz M, Thome C, Barth M, Harders A, Schmieder K (2011) Non-fusion rates in anterior cervical discectomy and implantation of empty polyetheretherketone cages. Spine (Phila Pa 1976) 36(1):15-20

33. Kasliwal MK, Baskin DS, Traynelis VC (2013) Failure of porous tantalum cervical interbody fusion devices: two-year results from a prospective, randomized, multicenter clinical study. J Spinal Disord Tech 26(5):239-245 